\title{
An Investigation of Range-velocity Deception Jamming Modeling
}

\author{
Xiong Ying, Gu Haiyan, Tang Bin, and Zhang Yongqiang \\ School of Electronic Engineering University of Electronic Science and Technology of China, Sichuan Province, China \\ Xiongy@uestc.edu.cn
}

\begin{abstract}
This paper explores the modeling method of rangevelocity deception jamming, which can deceive the victim radar on range and velocity information simultaneously. By analysis of the producing mechanism, maneuvering strategies and pulling rules of this jamming, the basic mathematical models are established by stages, as well as target echo and jamming models for pulse compression radar. Then based on the radar with LFM and Barker phase-coded signal, the specified models of the jamming are deduced. Simulation results show the received signal waveform when the target echo is presented in range-gate-pull-in and velocity-gatepull-in jamming (RGPI-VGPI) and range-gate-pull-off and velocitygate-pull-off jamming (RGPO-VGPO) environment. This will provide theoretical basis to Electronic countermeasures (ECM) and Electronic counter-countermeasures (ECCM) concerning rangevelocity deception jamming.

Index Terms - range-velocity deception jamming, active jamming, mathematical model.
\end{abstract}

\section{Introduction}

With the developing technology of the digital radio frequency memory (DRFM), range-velocity deception jamming is widely applied to interfering weapon-controlling radar, reconnaissance radar, warning and guidance radar owing to its rigorous logic and high similarity to the target echo ${ }^{[1]}$. When the jamming enters the radar receiver, the radar range and velocity gate will be simultaneously pulled off by time-delaying and frequency-shifting, resulting in the false target imitating the real target motion, which will directly affects the tactical decisions of the radar operators and even lead to radar losing the dominant position in battlefield. It is difficult for the conventional anti-jamming measures to counter range-velocity deception jamming, since the jamming can be produced through direct retransmission and enters the radar receiver mainly from the antenna main lobe ${ }^{[2]}$.

Using mathematical models of the jamming, the jammers are able to set up reasonable parameters to implement the jamming. on the other hand, the radar designers can develop more effective ECCM. Therefore, the investigation of rangevelocity deception jamming modeling is becoming increasingly important and will play a significant role both on ECM and ECCM in terms of the range-velocity deception jamming. This paper analyses the basic generation mechanism, the maneuver strategies and pull rules, and then establishes mathematical models of the target echo and the jamming by stages. This will provide theoretical basis to ECM and ECCM, laying a good foundation for jamming and anti-jamming.

\section{II . Generation mechanism of range-velocity jamming}

In order to more effectively interfere with radar, the waveforms transmitted by the deception jammer should match the radar waveforms. Usually, the implementation of the deception jammer divides into two categories, response type and repeat type. Due to the incoherent between responder interference and victim radar signal, modern deceptive electronic attack system often use DRFM to produce rangevelocity deception jamming whose signal is coherent with the radar transmitting signal. Thus, the jamming signals introduced into the coherent radar will obtain considerable processing gain (about $30 \mathrm{~dB}-60 \mathrm{~dB})^{[3]}$. The procedure to carry out the pull jamming on range-gate and velocity-gate of radar tracking system is as follows ${ }^{[4]}$ :

\section{A. Gate captured period}

The jamming pulses capture the range-gates. The jammer will retransmit the received radar pulse which has the same Doppler frequency with target echo in the minimum delay time. The typical delay time is $150 \mathrm{~ns}$ and the jamming pulse energy is greater than the target echo energy. This stage is called gate captured period and the duration is about $0.5 \mathrm{~s}-2 \mathrm{~s}$. The purpose of this stage is to make the jamming signal and target echo work on range-gates and velocity-gates simultaneously.

\section{B. Gate pulled period}

The jamming pulses pull the range-gates and velocitygates. The radar automatic gain control (AGC) circuit will be controlled by jamming pulses entering the tracking gate. Every time the jammer receive a radar pulse, it will gradually increase or decrease the delay time and adjust the Doppler frequency shift to make the range-gate and velocity-gate be away from the original gate gradually with the movement of the jamming pulses. The pull speed must be less than the maximum speed of the range-gate and velocity-gate's movement. The typical pull acceleration is about three times of the acceleration of gravity ${ }^{[3]}$.

\section{Gate-pulling stopped period}

The jammer stops transmitting jamming pulses at first and then repeats above-mentioned steps. When the gate is pulled far enough, the jammer stops transmitting jamming pulse. As a result, the target in the range-gate disappears suddenly, and the tracking system can only turn into the searching state. When 
the tracking system establishes a new range-gate and velocitygate, the jammer can consider the next gate pulling process.

During the gate pulled period, the jamming pulses are modulated by the delay time and Doppler frequency, causing range-gate and velocity-gate pull-in or pull-off in the radar receiver. Therefore, range-velocity deception jamming includes two subclasses, that is, RGPI-VGPI and RGPOVGPO. But for velocity-only radar and range-only radar, the jamming will display only one dimensional deception characteristic, that is, range-gate pull-off in range-only radar and velocity-gate pull-off in velocity-only radar.

\section{Maneuver analysis of range-velocity jamming}

Based on the relationship between the space positions of radar, target and jammer, the categories of jamming can be divided into stand-off jamming (SOJ), escort-screening jamming (ESJ), self-screening jamming (SSJ) and standforward jamming $(\mathrm{SFJ})^{[5]}$. Considering the security, rangevelocity active radar jamming excludes the SFJ basically. In addition, the ESJ hardly achieve ideal jamming effect because of lacking entrance to the radar antenna main lobe. As to the SSJ, jammer is located on the target, and the jamming can enter the radar receiver through the antenna main lobe, so the request for power is quite low, and then range-velocity deception jamming can be used as the SSJ in this case ${ }^{[6]}$. When the jammer has a higher transmitted power and is in the same radar angle resolution cell with the target, range-velocity deception jamming can also be used as the SOJ. The relative position relationship between radar and jammer is shown in Fig. 1 when range-velocity deception jamming is used as the SOJ and SSJ.

The radar transmitted signal is assumed as $s(t)$, and the distance between radar and SSJ is $r_{s}$ and $r_{o}$ between radar and SOJ.

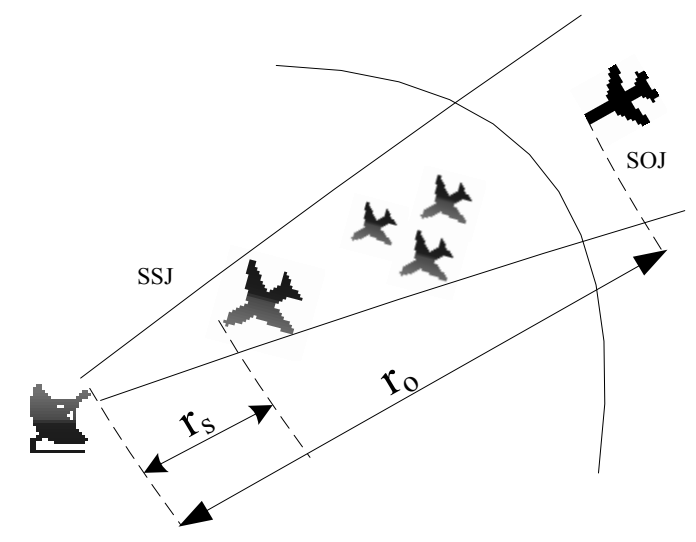

Fig. 1 Relative position relationship between radar, target and jammer.

The SOJ received signal can be approximated as $A_{s} s\left(t-r_{o} / C\right)$. In order to capture the range gate effectively and increase the range between the false target and the real target gradually, the jamming has to generate a relative delay time from zero to a considerable point increasingly. Therefore, if we adopt the SOJ, the jammer can only store the last period radar transmitted waveform and then retransmits it adding a certain delay modulation to offset the time-delay deviation $\left(r_{o}-r_{s}\right) / C$ produced by the range factors. From the analysis above, it is obvious to see that range-velocity deception jamming is restricted by the position of jammer and radar transmitted frequency or waveform agility et al when used as the SOJ. Meanwhile, the jamming generating process is relatively complicated because it has to offset the delay time produced by the range factors. So range-velocity deception jamming is mainly used as the SSJ.

\section{Basic mathematical models of range-velocity jamming}

Assume that the self-screening jammer (the target) moves with a velocity of $v_{r}$, and the distance between the jammer and the radar is $R(t)$. According to the echo model, the received target echo simplified model can be expressed as ${ }^{[7]}$

$$
S_{r}(t)=A_{r} s(t) \exp \left(j \omega_{r} t\right) \otimes \delta\left(t-\Delta t_{r}\right)
$$

where $A_{r}$ is the amplitude of the target echo, $\omega_{r}=2 \pi v_{r} / \lambda$ is the Doppler frequency shift caused by the target motion, and $\Delta t_{r}=2 R(t) / C$ is the target range delay time.

During the gate captured period, the intercepted radar signals by jammer are modulated by a certain amplitude $A_{j}$ and retransmitted in the minimum delay time $\Delta t_{d}$. In this case, the jamming and the target echo have the same Doppler frequency. Therefore, the mathematical model of the received range-velocity deception jamming by radar in this stage can be written as

$$
J(t)=A_{j} s(t) \exp \left(j \omega_{r} t\right) \otimes \delta\left(t-\Delta t_{r}-\Delta t_{d}\right)
$$

During the gate pulled period, the delay time and frequency shift of the jamming pulses change with the pull time $t^{\prime}$, and the received range-velocity active radar jamming by radar can be written as

$$
\begin{array}{r}
J(t)=A_{j} s(t) \exp \left(j\left(\omega_{,}+\omega_{j}\left(t^{\prime}\right)\right) t\right) \\
\otimes \delta\left(t-\Delta t_{r}-\Delta t_{d}-\Delta t_{j}\left(t^{\prime}\right)\right)
\end{array}
$$

where $A_{j}>A_{r}, \omega_{j}\left(t^{\prime}\right)=2 \pi \Delta f_{j}\left(t^{\prime}\right) . \Delta t_{d}$ is the jammer inherent delay time, and it is generally about $150 \mathrm{~ns}^{[8]}$. To imitate the target motion accurately, an internal relation between the time delay function $\Delta t_{j}\left(t^{\prime}\right)$ and the Doppler frequency shift function $\Delta f_{j}\left(t^{\prime}\right)$ of the range-velocity deception jamming should be satisfied, that is, in a certain time, the pull range reflected by the time delay function and the pull speed reflected by the Doppler frequency shift function should be matched. Assume that the velocity of the false target is $v_{j}$ 
when pulling off constantly, the corresponding range $\Delta R_{j}\left(t^{\prime}\right)$ of the time delay function can be expressed as

$$
\Delta R_{j}\left(t^{\prime}\right)=C \Delta t_{j}\left(t^{\prime}\right) / 2=v_{j} t^{\prime}
$$

So, when pulling off constantly, $\Delta t_{j}\left(t^{\prime}\right)$ and $\Delta f_{j}\left(t^{\prime}\right)$ have the relationship as follows,

$$
\begin{aligned}
& \Delta t_{j}\left(t^{\prime}\right)=2 v_{j} t^{\prime} / C \\
& \Delta f_{j}\left(t^{\prime}\right)=2 v_{j} / \lambda
\end{aligned}
$$

From (5), we can see that the Doppler frequency shift function $\Delta f_{j}\left(t^{\prime}\right)$ is a constant. In the radar system, the jamming delay time increases slowly relative to the real target delay time while the Doppler frequency has a step change which is easy to be identified by the radar. So it is unusual to use constant pulling method to generate range-velocity deception jamming. Assuming that the pulling acceleration is $a_{j}$ when the false target imitates uniformly accelerated motion, the corresponding range of the time delay function can be expressed as

$$
\Delta R_{j}\left(t^{\prime}\right)=C \Delta t_{j}\left(t^{\prime}\right) / 2=a_{j} t^{\prime 2} / 2 .
$$

So when pulling off with uniform acceleration, $\Delta t_{j}\left(t^{\prime}\right)$ and $\Delta f_{j}\left(t^{\prime}\right)$ have the relationship as follows,

$$
\begin{aligned}
& \Delta t_{j}\left(t^{\prime}\right)=a_{j} t^{\prime 2} / C \\
& \Delta f_{j}\left(t^{\prime}\right)=-2 a_{j} t^{\prime} / \lambda
\end{aligned}
$$

From (7), we can see that the Doppler frequency shift function $\Delta f_{j}\left(t^{\prime}\right)$ is time-variant when pulling with uniform acceleration. In radar system, the jamming delay time and Doppler frequency increase slowly with the pull time, and form a realistically mimetic target by making the velocity-gate and range-gate gradually get away from the original gates. Hence, pulling with uniform acceleration is the main method to generate range-velocity deception jamming. In addition, the jamming is regarded as RGPO-VGPO when $a_{j}>0$ while RGPI-VGPI when $a_{j}<0$, and the pull range increases gradually in the former case while decreases in the latter.

\section{Specified models of range-velocity jamming}

With large time-bandwidth product, the pulse-compressed signal has not only good performance in detection but also high range resolution and velocity resolution. The signals which are modulated by intra-pulse frequency or phase, like the LFM signal and Barker phase-coded signal, are widely used in modern radar system.

\section{A. Jamming Model of the LFM Radar}

When the radar transmitted signal is the LFM pulse signal, it can be assumed to be

$$
s(t)=\operatorname{Arect}(t / T) \exp \left(j\left(\omega_{0} t+\mu t^{2} / 2+\phi\right)\right)
$$

where $A$ is the amplitude; $\operatorname{rect}(t / T)=1, \quad|t| \leq T / 2$ and $\operatorname{rect}(t / T)=0, \quad|t|>T / 2$ is the rectangle function; phase $\phi$ obeys uniform distribution in the region $[0,2 \pi] ; \omega_{0}$ is start frequency; $\mu$ is the chirp rate, and $T$ is the pulse width. According to (1), the radar received target echo can be expressed as

$$
\begin{aligned}
S_{r}(t)= & A_{r} \operatorname{rect}(t / T) \exp \left(j \left(\left(\omega_{f}+\omega_{\gamma}\right) t\right.\right. \\
& \left.\left.+\mu t^{2} / 2+\phi\right)\right) \otimes \delta\left(t-\Delta t_{r}\right)
\end{aligned}
$$

Substituting (9) into (2) and (3), the jamming model can be expressed as

$$
J(t)=\left\{\begin{array}{l}
A_{\mathrm{j}} \operatorname{rect}(t / T) \exp \left(j\left(\left(\omega_{0}+\omega_{\mathrm{r}}\right) t+\mu t^{2} / 2+\phi\right)\right) \\
\otimes \delta\left(t-\Delta t_{\mathrm{r}}-\Delta t_{\mathrm{d}}\right) \text { in gate captured period } \\
A_{\mathrm{j}} \operatorname{rect}(t / T) \exp \left(j\left(\left(\omega_{0}+\omega_{\mathrm{r}}+\omega_{\mathrm{j}}\left(t^{\prime}\right)\right) t+\mu t^{2} / 2+\phi\right)\right) \\
\otimes \delta\left(t-\Delta t_{\mathrm{r}}-\Delta t_{\mathrm{d}}-\Delta t_{\mathrm{j}}\left(t^{\prime}\right)\right) \text { in gate pulled period }
\end{array}\right.
$$

\section{B. Jamming Model of the Barker Phase-coded Radar}

The modulation function of the LFM signal is continuous in a finite domain, while the modulation function of phasecoded signal is discrete in a finite state ${ }^{[9]}$. The Baker phasecoded signal is generally given by

$$
\begin{aligned}
s(t)= & A \sum_{i=1}^{N} \operatorname{rect}\left(\left(t-(i-1) T_{d}\right) / T\right) \\
& \exp \left(j\left(\omega_{0} t+\varphi(t)+\varphi_{0}\right)\right)
\end{aligned}
$$

where $A$ is the amplitude, $N$ is the number of code elem ent, $T_{d}$ is the width of code element, $\omega_{0}$ is the carrier frequen cy, $\varphi_{0}$ is the initial phase, and $\varphi(t)$ is the phase modulation $\mathrm{f}$ unction. The rule of phase modulation is when the code is 0 , th e phase takes 0 and when the code is 1 , the phase takes $\pi^{[10]}$. Assuming that the radar transmits signal which is $s(t)$, then ta rget echo received by radar is given by

$$
\begin{aligned}
S_{\mathrm{r}}(t)= & \sum_{i=1}^{N} A_{r} \operatorname{rect}\left(\left(t-(i-1) T_{d}\right) / T\right) \\
& \lessdot \exp \left(j\left(\omega_{0} t+\varphi(t)+\varphi_{0}\right)\right) \otimes \delta\left(t-\Delta t_{\mathrm{r}}\right)
\end{aligned}
$$


where $\omega_{r}$ is the Doppler frequency shift caused by target motion, and $\Delta t_{r}$ is the time delay caused by the target motion.

During the wave gate captured period, the radar received active range-velocity $\mathrm{SSJ}$ has a constant delay time $\Delta t_{d}$ relative to the target echo, and the jamming model can be expressed as

$$
\begin{aligned}
J(t)= & \sum_{i=1}^{N} A_{\mathrm{j}} \operatorname{rect}\left(\left(t-(i-1) T_{d}\right) / T\right) \\
& {\left[\exp \left(j\left(\left(\omega_{\mathrm{o}}+\omega_{\mathrm{r}}\right) t+\varphi(t)+\varphi_{0}\right)\right) \otimes \delta\left(t-\Delta t_{\mathrm{r}}-\Delta t_{\mathrm{d}}\right)\right.}
\end{aligned}
$$

During the wave gate pulled period, the waveform received by radar is frequency-modulated or time-delaymodulated by the jammer. Where the jamming model can be expressed as ${ }^{[11]}$

$$
\begin{aligned}
J(t)= & \sum_{i=1}^{N} A_{\mathrm{j}} \operatorname{rect}\left(\left(t-(i-1) T_{d}\right) / T\right) \\
& \exp \left(j\left(\left(\omega_{\mathrm{o}}+\omega_{\mathrm{r}}+\omega_{\mathrm{j}}\left(t^{\prime}\right)\right) t+\varphi(t)+\varphi_{0}\right)\right) \\
& \otimes \delta\left(t-\Delta t_{\mathrm{r}}-\Delta t_{\mathrm{d}}-\Delta t_{\mathrm{j}}\left(t^{\prime}\right)\right)
\end{aligned}
$$

\section{Simulation}

\section{A. Case one}

The radar transmits the LFM pulse signal whose wavelength is $0.01 \mathrm{~m}$, and its bandwidth is $20 \mathrm{MHz}$, and its product of time and bandwidth is 50 , and the jamming power gain is $A_{j} / A_{r}=3 / 2$. Its pull acceleration is $3 g$, and its duty-cycle is $1 / 15$. The target has a constant speed of $200 \mathrm{~m} / \mathrm{s}$ when it is $2 \mathrm{~km}$ away from the radar. In each jamming period, the LFM radar received the RGPI-VGPI and RGPO-VGPO is shown in Fig.2.
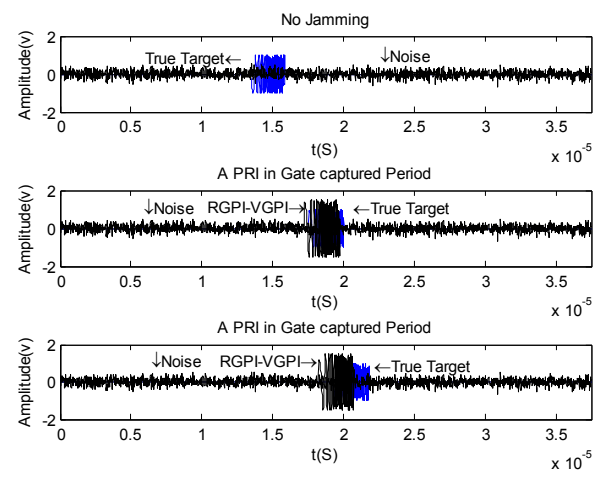

(a) RGPI-VGPI with $\mathrm{SNR}=15 \mathrm{~dB}$.
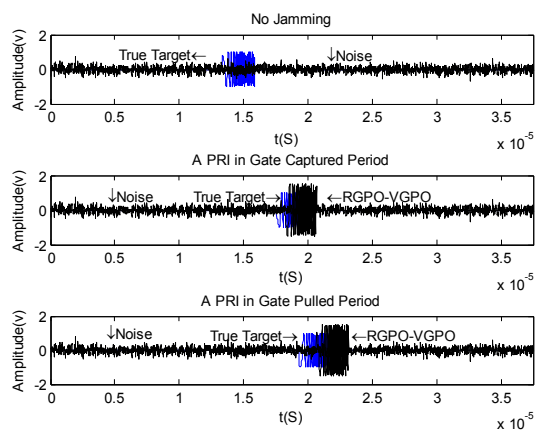

(b) RGPO-VGPO with $\mathrm{SNR}=15 \mathrm{~dB}$.

Fig. 2 Zero-IF signal waveform received by the LFM radar.

As shown in Fig.2, when range-velocity deception jamming is the RGPI-VGPI, the jamming will arrive at the receiver before the target echo. On the contrary, the jamming will arrive at the receiver after the target echo when it is the RGPO-VGPO. During the gate captured period, the jamming and target echo in radar received waveform basically overlap. While during a pulse repeated interval in the gate pulled period, the overlapping part of the jamming and target echo reduces and it indicates that the wave gate has been away pulled off the original position gradually.

\section{B. Case two}

The radar transmits 13 bits Barker phase-coded pulse signal whose wavelength is $0.01 \mathrm{~m}$, and its time width is $2.5 \mu \mathrm{s}$, and its jamming power gain is $A_{j} / A_{r}=3 / 2$. Its pull acceleration is $3 g$, and its duty-cycle is $1 / 15$. The true target has a constant speed of $200 \mathrm{~m} / \mathrm{s}$ when it is $2 \mathrm{~km}$ away from the radar. In each jamming period, the Baker radar received the RGPI-VGPI or RGPO-VGPO is shown in Fig.3. Therefore, we can obtain that during the gate pulled period, the overlapping part of the jamming and target echo obviously reduces, while the jamming and target echo almost overlap during the gate captured period, no matter it is either the RGPI-VGPI or RGPO-VGPO. Moreover, as the gate-pulling time increases, the relative delay time of jamming to the target echo will increase gradually which can achieve the goal of being away from the original gate.
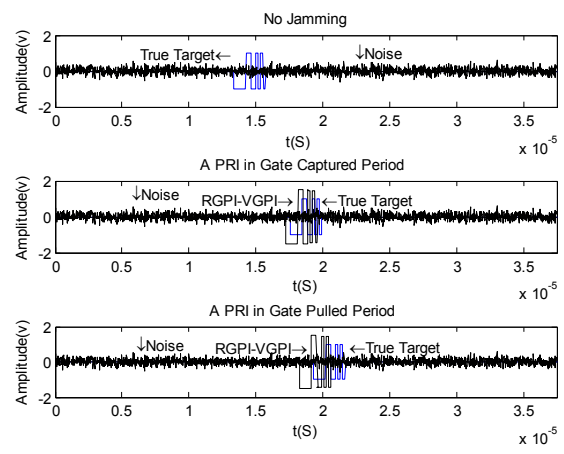

(a) RGPI-VGPI with SNR $=15 \mathrm{~dB}$. 

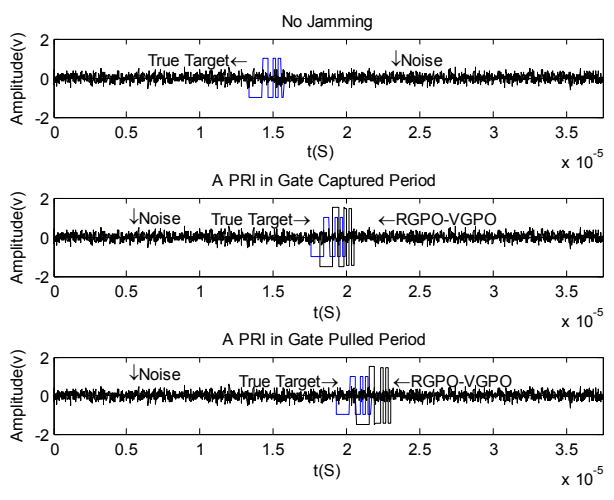

(b) RGPO-VGPO with $\mathrm{SNR}=15 \mathrm{~dB}$.

Fig. 3 Zero-IF signal waveform received by the Barker radar.

\section{Conclusion}

Range-velocity deception jamming has brought about a serious threat to modern radar. The paper analyses the jamming generation mechanisms, then discusses the proper type of actual operation and pull rules of this kind of jamming, and establishes the mathematical models based on jamming procedure. Finally, we plot receiving zero-IF signal waveform in each stage of range-velocity deception jamming, which provides theoretical basis for the research of range-velocity deception jamming and anti-jamming.

\section{References}

[1] Schuerger J., Garmatyuk D., "Deception jamming modeling in radar sensor networks," Military Communications Conference, 2008. MILCOM 2008. IEEE, vol., no., pp.1, 7, 16-19, Nov. 2008.

[2] Greco M., Gini F., Farina Alfonso, "Radar Detection and Classification of Jamming Signals Belonging to a Cone Class," Signal Processing, IEEE Transactions on, vol.56, no.5, pp.1984-1993, May 2008.

[3] D. C. Schleher, Electronic warfare in the information age, Massachusetts: Artech House, Norwood, 1999.

[4] Fan Wei, Research on the feature extraction and identification algorithm of radar active jamming, Master thesis, Chengdu: UESTC, 2007.

[5] Zhao Guoqing, The principle of radar ECM, Xi'an: Xidian University press, 1999.

[6] Zhang Yi, Radar jamming modeling and simulation, Master thesis, Xi'an: Xidian University, 2009.

[7] Sun Minhong, Research of active deception radar jamming suppression, Doctoral thesis, Chengdu: UESTC, 2008.

[8] Zhang Mingyou, Wang Xuegang, Radar system, Beijing: electronic industry press, 2006.

[9] Li Jifeng, Sheng Jisong, "Study of Phase-coded Radar Jamming Technology," Modern Electronics Technique, vol.21, no., pp.17-24, Nov. 2009.

[10] Yang Wanhai, Modeling and Simulation for Radar System, Xi'an: Xidian University press, 2007.

[11] Gu Haiyan, Research on jamming modeling and countermeasures of range-velocity active radar, Master thesis, Chengdu: UESTC, 2011. 